\title{
Octreotide therapy: a new horizon in treatment of iatrogenic chyloperitoneum
}

\author{
C Bhatia, U Pratap, Z Slavik
}

\begin{abstract}
Chyloperitoneum is a rare and challenging complication of abdominal surgery. We report a case of iatrogenic chyloperitoneum. Infusion of octreotide, a somatostatin analogue, together with total parenteral nutrition followed by medium chain triglyceride diet resulted in rapid resolution of chyloperitoneum. We believe this to be the first report of successful use of octreotide in iatrogenic chyloperitoneum in a child.

(Arch Dis Child 2001;85:234-235)
\end{abstract}

Keywords: chyloperitoneum; octreotide; abdominal surgery

Chyloperitoneum is an infrequent complication following intra-abdominal surgery. ${ }^{1}$ Its management is challenging as a result of the usually chronic course, difficulty in localisation of its source, and lack of availability of effective treatment. $^{2}$ Conservative treatment of chyloperitoneum involves a medium chain triglyceride (MCT) based diet or total parenteral nutrition (TPN). Surgery is recommended when conservative treatment fails. We report the successful use of octreotide in the treatment of chyloperitoneum in a 5 year old girl following laparotomy for duodenal bleeding.

\section{Case report}

A 5 year old girl, who had received an orthotopic cardiac transplant for dilated cardiomyopathy two years earlier was admitted to our unit with severe acute rejection. She was treated with methylprednisolone followed by recombinant antithymocytic globulin. On day 21 of admission she developed massive gastrointestinal bleeding with melaena, which did not resolve with ranitidine, omeprazole, and blood

Accepted 14 May 2001

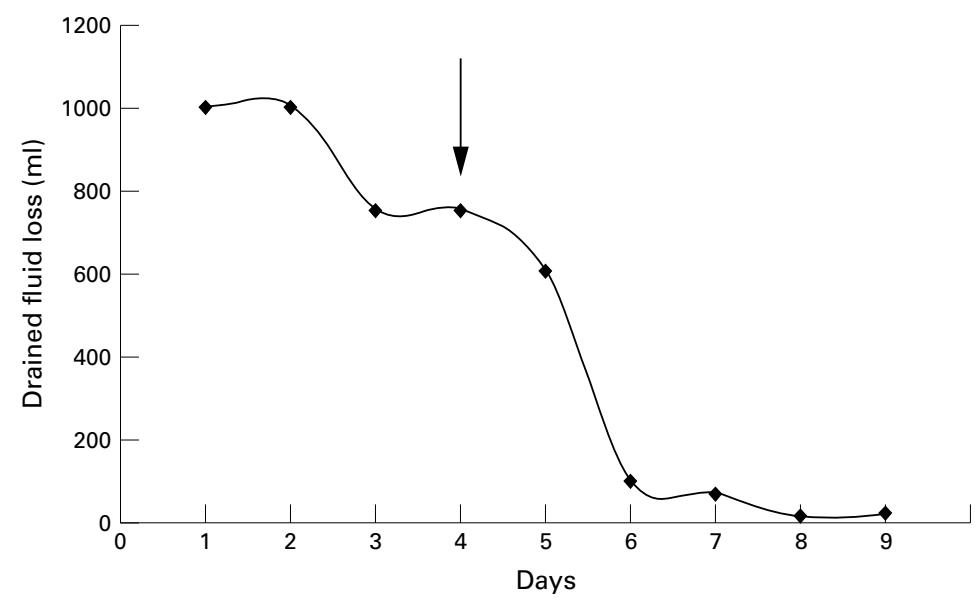

Figure 1 Reduction in drained fluid losses following the start of octreotide therapy (arrow). transfusions. Laparotomy and endoscopically assisted duodenotomy was performed on day 31. Four bleeding points were identified and sutured. She made good progress following this procedure and was discharged on day 62 .

She was readmitted two months later with another episode of acute rejection despite a standard triple immunosupression regime. During this admission she developed Clostridium difficile enteritis for which she was treated with vancomycin and TPN. Abdominal distension was noted on day 12 of admission. Abdominal ultrasound examination confirmed ascites; abdominal paracentesis on day 17 revealed cloudy fluid. Biochemical analysis confirmed a diagnosis of chylous ascites with chylomicrons; cholesterol and triglyceride concentrations were $1.9 \mathrm{mmol} / \mathrm{l}$ and $5.7 \mathrm{mmol} / \mathrm{l}$, respectively. A significant volume of chylous fluid was expressed through the abdominal drain over the following days (0.7-1 1/day; fig 1$)$ despite continued TPN. Serum albumin concentrations stayed above $37 \mathrm{~g} / 1$ with colloid replacement treatment containing fresh frozen plasma and albumin solutions. Octreotide (Sandostatin, Novartis Pharmaceuticals UK Ltd) was started four days after chylous drainage. The intravenous infusion was increased from $1 \mu \mathrm{g} / \mathrm{kg} / \mathrm{h}$ to $2 \mu \mathrm{g} / \mathrm{kg} / \mathrm{h}$ on day 3 of treatment. Drained losses significantly decreased from $1000 \mathrm{ml} /$ day to $13 \mathrm{ml} /$ day within three days of treatment (fig 1). The peritoneal catheter was removed on day 5 of treatment and octreotide infusion was stopped three days later. She made a good recovery without recurrence of ascites. She was discharged from hospital on an MCT diet 12 days after the diagnosis of chyloperitoneum. Follow up assessment two months later did not show any recurrence of chyloperitoneum while on a normal diet.

\section{Discussion}

Congenital chylous ascites is the commonest cause of chyloperitoneum in young children. Other causes of chylous ascites in children are either idiopathic or obstructive lesions caused by malrotation, intussusception, incarcerated hernia, lymphangioma, blunt trauma, liver disease, and tuberculosis. ${ }^{2}$ Chylous ascites is an infrequent yet alarming complication of intraabdominal surgery. ${ }^{1}$ It is not benign; a mortality rate of $17 \%$ was reported in the most recent meta-analysis of paediatric cases. ${ }^{3}$ Our patient developed clinically significant chylous ascites 2.5 months after her laparotomy. This delayed presentation could be explained by the relatively slow return of her appetite postoperatively, together with the increased central venous pressure related to the recurrence of her acute cardiac rejection. There was no evidence 
of any of the above mentioned non-iatrogenic causes of chylous ascites in our patient before, at the time of, or after her abdominal surgery.

Management of chylous ascites is controversial. Modalities available for the treatment are diet or enteric rest with TPN, with or without drainage of the fluid. The aim of the MCT diet is to reduce lymph flow and to allow the chylous fistula to heal naturally. However, Unger and Chandler ${ }^{2}$ did not observe any benefit of an MCT diet for chylous ascites. Others have shown only a partial effect (36\% success rate) over four to six months of dietetic treatment. ${ }^{3}$ TPN negated the risk of nutritional deficiency and, since it reduced accumulation of chylous fluid in the peritoneal cavity, it also obviated any loss of protein and fat following repeated paracenteses. ${ }^{2}$ A satisfactory outcome of TPN in chyloperitoneum required at least three to four weeks of treatment. ${ }^{3}$ Surgical ligation of the chylous fistula is recommended when conservative treatment fails. ${ }^{2}$ Browse and colleagues ${ }^{4}$ reviewed 30 patients aged 1 month to 17 years who were treated surgically, achieving total cure in $33 \%$ and notable improvement in a further $36 \%$. The remaining patients had poor results of their surgical treatment with $77 \%$ mortality.

Given the limitations of the existing treatment modalities, the use of octreotide, a synthetic analogue of somatostatin with multiorgan effect, has been considered. ${ }^{5}$ Octreotide decreases intestinal blood flow and inhibits lymph secretion through somatostatin receptors in the intestinal wall, thereby improving chylous ascites. There is only one previously published case report on its successful use in an infant with congenital chylous ascites. ${ }^{6}$ The successful outcome was achieved by the combined use of subcutaneous octreotide, $\mathrm{TPN}$, and an MCT diet. In our case the aetiology of chyloperitoneum was iatrogenic. We have used the intravenous infusion with comparable effect on chylous drainage. No significant side effects were noted. Our patient was discharged within 12 days of diagnosis and ascites did not recur.

To our knowledge this is the first case report of the successful use of octreotide in iatrogenic chylous ascites in a child. Octreotide represents a promising adjunct to the conservative treatment of chylous ascites in children; the recent development of a long acting somatostatin analogue could make treatment of this rare condition even easier.

1 Ablan CJ, Littooy FN, Freeark RJ. Post-operative chylous ascites: diagnosis and treatment. Arch Surg 1990;125:270-3. Unger SW, Chandler JG. Chylous ascites in infants and children. Surgery 1983;93:455-61.

3 Man DKW, Spitz L. The management of chylous ascites in children. F Pediatr Surg 1985;20:72-5.

4 Browse NL, Wilson NM, Russo F, et al. Etiology and treatment of chylous ascites. Br $\mathcal{F}$ Surg 1992;79:1145-50.

5 Reubi JC, Horisberger U, Waser B, et al. Preferential location of somatostatin receptors in germinal centers of location of somatostatin receptors in germinal centers of
human gut lymphoid tissue. Gastroenterology 1992;103: human $1207-14$.

6 Caty MG, Hilfiker ML, Azizkhan RG, Glick PL. Successful

Caty MG, Hilfiker ML, Azizkhan RG, Glick PL. Successful
treatment of congenital chylous ascites with a somatostatin analogue. Pediatr Surg Int 1996;11:396-7.

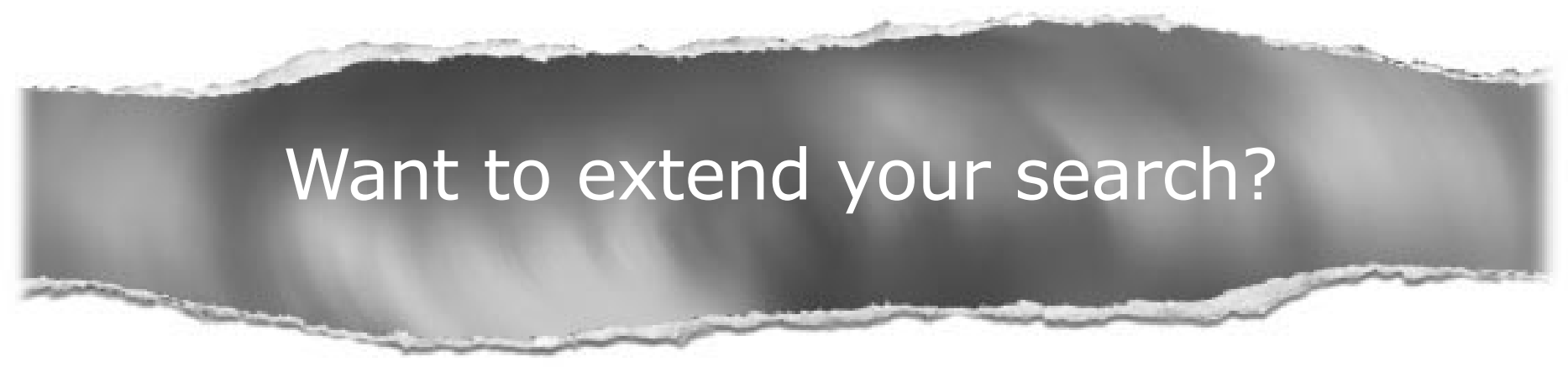

Cross journal searching

If you can't find what you are looking for in Archives of Disease in Childhood you can extend your search across many of the more than 200 journals available for selection. You can restrict your search to specific subject areas (eg, clinical medicine, basic research), or select specific journals, or search all available titles.

www.archdischild.com 